\title{
ON THE SOLUTION OF DIFFICULTLY-SOLUBLE SUBSTANCES.
}

*By Alfred H. Alien, F.C.S.

Some years ago, in a letter to the Chemical News, (Vol. XXII., p. 57), I described a mode of effecting the solution of difficultly-soluble iron ores and slags, by heating them with strong hydrochloric acid in sealed tubes, and experience having shown the method to be extremely valuable in certain cases, I have thought it worth while to bring my results before the Society in the form of a paper.

In the analysis of minerals and metallurgical products containing iron, it is often necessary to determine whether that metal exists as a ferrous or a ferric compound. Of course, this is easy, provided the substance is readily decomposed by acids, but in the case of insoluble or difficultly-soluble minerals and slags, the problem is by no means easy of solution. Protracted boiling with acid is often very inconvenient, and sometimes useless; while any process of fusion almost necessarily involves more or less oxidation of ferrous compounds.

Mr. C. E. Avery (Chemical News, Vol. XIX., p. 270), has proposed to decompose silicates with a mixture of a fluoride and a mineral acid, and the same method in a somewhat modified form has been described by Messrs. Wilbur \& Whittlesey (Chemical News, Vol. XXII., p. 2). These processes have a certain value of their own, but the use of fluorine compounds is not always convenient, though of course in some cases indispensable.

It might be anticipated that acids acting under pressure in sealed tubes would effect the decomposition of many refractory minerals, which resist ordinary methods of treatment, and this view is fully borne out by experience. This mode of treatment has the great advantage that it can be continued for any desired length of time, or discontinued and recommenced at will, and that the subsequent steps of the analysis can be performed after any interval of time most convenient to the operator.

The method of procedure I have been in the habit of adopting is simply as follows : A gramme of the finely powdered mineral or slag is placed in a piece of combustion tube carefully sealed at one end. From 20 to 30 c.c. of pure fuming hydrochloric acid are poured in, and the other end of the tube drawn out and carefully sealed in the blowpipe flame. The tube when sealed, should be about eight or ten inches in length. The proportion of acid used is such as to ensure a large excess; this prevents undue weakening by saturation of the acid, and has other obvious advantages. 
The tube is now ready for heating. In many cases, the mere heat of a water-bath is amply sufficient to ensure perfect decomposition. As fuming acid is employed, there is always considerably more than one atmosphere of pressure even at $100^{\circ} \mathrm{C}$, but decomposition is in many cases greatly facilitated by use of a higher temperature. A very convenient bath for the purpose is formed by a saturated solution of nitrate of sodium, which boils at $120^{\circ} \mathrm{C}$. In some cases, it is desirable to obtain a still higher temperature, in which case chloride of calcium can be conveniently employed. In all cases, however, I prefer to subject the tube to a temperature not exceeding $100^{\circ} \mathrm{C}$. first of all. This enables the sealing to be tested, and perhaps causes some reduction of the internal pressure, owing to partial neutralization of the acid. In many cases the temperature of $100^{\circ} \mathrm{C}$, is amply sufficient to effect perfect decomposition of the sample.

This is true of blast-furnace slag, all the rarieties of which are readily decomposed at $100^{\circ} \mathrm{C}$. In fact, blast-furnace slag rarely requires a sealed tube at all. The slag now obtained in England from the manufacture of spiegeleisen, contains from 30 to 40 per cent. of oxide of manganese, and is decomposed with such facility that if it be added in a powdered state to hot hydrochloric acid, the mixture will be converted, almost instantly, into a transparent jelly.

"Tap-cinder" and Bessemer-converter slag are far more refractory, and require somewhat prolonged treatment at an elevated temperature.

Basalt is readily and completely decomposed when heated with fuming hydrochloric acid in a sealed tube.

Titanic iron sand, if finely powdered, suffers perfect decomposition under the same treatment.

"Ore-furnace slag" from copper-smelting, is decomposed with difficulty.

Finely powdered burnt red brick, gives up some iron to the acid, but is very imperfectly decomposed.

Chrome-iron ore suffers very little change.

Tin-stone is partially dissolved, but I have not succeeded in effecting perfect solution.

A blank experiment showed that the acid had no sensible effect on the glass of the tube, even when heated in it to about $169^{\circ} \mathrm{C}$. for five hours.

In all cases it is exceedingly easy to watch the progress of the decomposition. When complete, the tube can be left alone till it is convenient to proceed with the analysis. For the determination of the ferrous oxide, it is only necessary to open the tube, wash the contents into a basin, and titrate at once with standard bichromate.

The silica rarely separates in a gelatinous state.

It has been proposed to employ a mixture of three parts by weight of concentrated sulphuric acid, and one of water, for a similar purpose. Experiment shows that this is by no means a satisfactory modification, at least for the treatment of ferruginous silicates and similar materials, the sulphate of iron separated preventing further action on the undecomposed portions of the substance. In fact, I have succeeded in effecting complete decomposition by sulphuric acid in comparatively few cases.

It is evident that treatment in sealed tubes is not at all an advantageous plan of employing sulphuric acid, for unless the temperature be dangerously high, the vapour of 
the acid exerts no great tension, and thus all the advantages of working under high pressure are lost, and one might as well use an ordinary bottle or corked tube at once.

But at very high temperatures sulphuric acid is able to effect decompositions with great facility, and, at a red heat, it is one of the most convenient and powerful re-agents we possess.

Of course "red-hot sulphuric acid" is employed in the form of acid sulphate of potassium. The sodium salt is often recommended, but it will not adrantageously replace the potassium compound, as it decomposes at an inconveniently low temperature, and gives off much more sulphuric anhydride.

The acid sulphate should always be fused in the platinum crucible first of all, to drive off water and free acid, the sample being subsequently added.

It is desirable to test the purity of any fresh sample of " bisulphate" before using it. A quantity of the salt sold me by one of our principal operating ehemists contained a considerable quantity of silver, an impurity doubtless dissolved from the ressel in which the salt had been fused by the manufacturer.

By fusion with acid sulphate of potassium, nearly all the above-mentioned refractory substances can be readily decomposed. Brick-dust leaves nothing but pure white silica, and the same is true of all clays.

Instead of treating ordinary soluble iron ores with hydrochloric acid, and having the unsatisfactory item of "silicious matter" to examine further, it is far better to fuse the ore with acid sulphate at once, by which means pure white insoluble silica is obtained, and all the other constituents pass into solution on treating the product of the fusion with acidulated water. In presence of pyrites or ferrous compounds it is best to add a crystal of nitre when the sample is placed in the crucible. The silica obtained is sometimes ochreous if too high a temperature has been employed, but it is readily purified by treatment with hydrochloric acid after pouring off the liquid. No evaporation to dryness to render the silica insoluble is requisite.

It is a pity that a re-agent so valuable and so widely applicable as the acid sulphate of potassium is not more commonly employed.

I have made a few experiments in another direction, and although the results possess merely a negative value, this appears a convenient occasion to place them on record.

It is well known that a hot solution of phosphoric acid readily acts on glass, and it occurred to me that the decomposing power would probably be greatly enhanced if fused meta-phosphoric acid were employed instead of a mere solution. This deduction was fully borne out by experiment. A fragment of window-glass about an inch square was treated at a low red heat in a platinum crucible with glacial phosphoric acid. In an hour or so it was decomposed nearly to the centre, and the change was accompanied by some very remarkable and characteristic appearances. With powdered glass the reaction was still more perfect, and there is no doubt that fusion with phosphoric acid might be employed as a means of decomposing silicates. The difficulty consists in the subsequent treatment of the resulting meta-phosphates. In practice, this presents such difficulties, that I have been compelled to abandon the idea of making the method useful, though it is possible that there are exceptional cases in which it might be made of service. The platinum crucible used for the fusion is seriously attacked. 